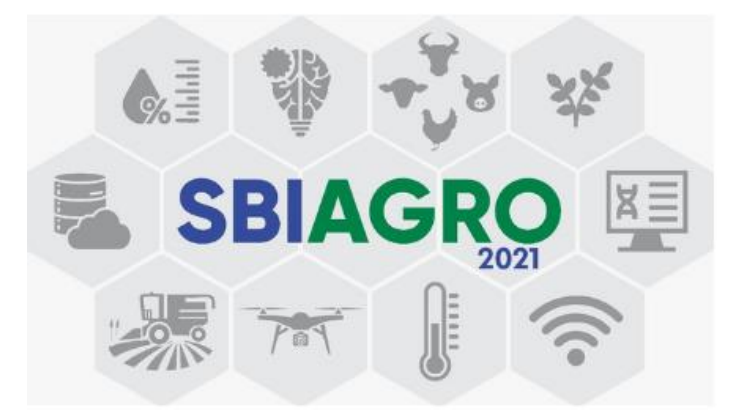

\title{
Desenvolvimentos em inteligência artificial na avicultura de frangos de corte
}

\author{
Lucas Gabriel Galdino da Silva ${ }^{1,2}$, Francisco Gomes Cordeiro Neto ${ }^{1}$, Josenalde \\ Oliveira $^{1,2}$, Laura Santana ${ }^{1,2}$ \\ ${ }^{1}$ Escola Agrícola de Jundiaí - Universidade Federal do Rio Grande do Norte (UFRN) \\ Macaíba - RN - Brasil \\ ${ }^{2}$ Instituto de Engenharia de Sistemas, Computadores, Pesquisa e Desenvolvimento do \\ Brasil (INESC P\&D Brasil) \\ Santos - SP - Brasil \\ \{lucas.gabriel.silva.706, francisco.neto.009\}@ufrn.edu.br, \\ \{josenalde.oliveira, laura.santana\}@ufrn.br
}

\begin{abstract}
The Brazilian poultry industry has been gaining prominence as a major producer and occupies the top position in the world ranking. In this review, based on an exploratory survey, there are presented research outcomes with Artificial Intelligence (AI) throughout the production chain in broiler poultry farming, from behavior analysis for disease identification and slaughter weight prediction, to quality control. With a period beginning with the international release of Industry 4.0, there are described 26 papers which demonstrate the growing adoption of AI and its divisions.
\end{abstract}

Resumo. A indústria avícola brasileira vem ganhando destaque como grande produtor e ocupa o topo de colocações no ranking mundial. Na presente revisão, a partir de um levantamento exploratório, são apresentados resultados de pesquisas que utilizem Inteligência Artificial (IA) ao longo da cadeia de produção de frangos de corte, desde a análise de comportamentos para identificação de doenças e predição de peso de abate, ao controle de qualidade. Fazendo o período de estudo coincidir com o início internacional da Indústria 4.0, são relatados 26 trabalhos que apontam para a adoção crescente da IA e suas divisões. 


\section{Introdução}

A cadeia produtiva de frangos de corte é uma das cadeias mais importantes no agronegócio brasileiro (BASSI; SILVA, 2017), o que é corroborado ao analisar-se os dados de produção e exportação desse setor. De acordo com relatório 2021 da Associação Brasileira de Proteína Animal (ASSOCIAÇÃO BRASILEIRA DE PROTEÍNA ANIMAL, 2021), em 2020 o Brasil produziu 13,845 milhões de toneladas de carne de frango e exportou 4,231 milhões de toneladas, o que torna o país o maior exportador mundial do produto, sendo que dessa exportação, 66,99\% é do produto tipo cortes, com frango inteiro, embutidos, salgados e industrializados complementando este percentual. No estudo feito por Bassi e Silva (2018), os autores afirmam que os grandes avanços da pesquisa no desenvolvimento de novas tecnologias no setor da avicultura ajudaram a posicionar o Brasil em termos de produção e exportação mundial. Nesse contexto, é interessante observar que a agroindústria, assim como a indústria de modo geral, vem passando por um processo de revolução tecnológica conhecido como Indústria 4.0, onde as chamadas tecnologias habilitadoras 4.0, dentre elas a Inteligência Artificial (IA), permitem não apenas otimizar e monitorar processos, mas também auxiliar na tomada de decisão, estando usualmente integrada às camadas de modelagem supervisionada e não supervisionada em algoritmos de ciência de dados.

No caso do agronegócio, tem sido comum adotar genericamente o termo Agricultura 4.0 e nesse caso, tais tecnologias também fomentam a agricultura de precisão, na qual busca-se adotar "estratégias de manejo mais eficientes, considerando a variabilidade espacial e temporal da unidade produtiva, permitindo o uso adequado dos insumos a fim de evitar desperdícios" (SANTANA; OLIVEIRA, 2021). É comum a adoção dos termos inteligente ou smart para diferenciar da prática convencional. $\mathrm{Na}$ produção animal, a zootecnia de precisão tem se ocupado de questões "para supervisão e análise em tempo real da saúde, nutrição, comportamento e ambiência, visando os índices produtivos, reprodutivos e bem-estar animal de forma automática, contínua, não invasiva e sem gerar estresse ao animal" (ROCHA; SILVA, 2020). O tema tem, inclusive, motivado a ampliação de chamadas públicas, como o Programa Agro 4.0 da Agência Brasileira de Desenvolvimento Industrial (ABDI), cujo $1^{\circ}$ edital selecionou e premiou 14 projetos pilotos de adoção e de difusão de tecnologias 4.0 no agronegócio (BRASIL, 2020a). Na mesma linha seguiu a Financiadora de Estudos e Projetos (FINEP), com chamada de subvenção econômica às empresas em tecnologias 4.0, incluindo o setor agropecuário (BRASIL, 2020b).

Devido ao volume, variedade e velocidade de dados disponíveis para geração de conhecimento, a IA tem sido muito explorada na atualidade em soluções para cidades, saúde e indústria. Não seria diferente para o setor agropecuário, onde a IA encontra adequação natural à complexidade de mapeamento das relações biológicas e de ambiência. Diante do exposto, este artigo tem como objetivo fazer um levantamento exploratório inicial sobre como a IA tem sido adotada na avicultura de frango de corte no período que configura o início do conceito Indústria 4.0, destacando as principais aplicações e identificação de demandas para o avanço da área a partir desta tecnologia habilitadora. 


\section{Material e Métodos}

Com base na metodologia PRISMA para organização de revisões bibliográficas (MOTHER et al., 2009), para a etapa de identificação, foi utilizada a busca geral por assunto no Portal de Periódicos CAPES, com acesso federado via Cafe/RNP, com a string ("machine learning" OR "artificial intelligence") AND (broiler OR poultry OR chicken OR aviculture). Dado que a busca contempla por padrão os campos Título, Resumo, Abstract, Palavras-Chave, o uso da string com termos em língua inglesa também contempla as principais bases indexadoras (Springer, IEEE, Science Direct) e periódicos nacionais. De 4.176 trabalhos, são apresentados o total de 26 estudos, após aplicação dos critérios de inclusão: período de 2016-2021, avicultura de frango de corte, trabalhos completos indexados e revisados por pares. Adicionalmente, os critérios de exclusão foram aplicados: avicultura de postura, ênfase em Internet das Coisas (Internet of Things, IoT), não frango, e com conteúdo não diretamente relacionado, além da remoção de duplicados. A busca com termos em português: ("inteligência artificial" OR "aprendizagem de máquina") AND (frango OR avicultura) não retornou resultados.

Embora trabalhos com IoT apresentem intersecções e possam contemplar etapas adicionais de análise e tratamento dos dados, revisões específicas para o aspecto de instrumentação podem ser encontradas em Oliveira et al. (2019) e Astill et al. (2020). O período dos últimos cinco anos foi selecionado de modo a coincidir com a promoção do termo Indústria 4.0 a partir do trabalho de Schwab (2016) e sua relação com IA.

\section{Resultados e Discussão}

Dos 26 estudos listados, usando o critério de afiliação do primeiro autor e instituição de condução da pesquisa, o Brasil encontra-se em 2. lugar, com 3 trabalhos, atrás apenas da China, com 6 trabalhos. Os demais artigos distribuem-se pela Ásia, América do Norte e Europa. A representação brasileira inclui os estados de São Paulo e Paraná (RIBEIRO et al., 2019; BARBON et al., 2018; PEREZ et al., 2018), com instituições como Unicamp, USP, UEL, UFPR, UTFPR e PUC-PR.

Da gestão e manejo das condições de ambiência em aviários para aprimoramentos no bem-estar animal (RIBEIRO et al., 2019), seleção genética de matrizes, produtividade e detecção antecipada de enfermidades, ao processamento agroindustrial em suas etapas de controle de qualidade, o melhor conhecimento do processo por meio da análise automatizada dos dados de produção baseada em IA, maximiza a assertividade da tomada de decisão e o cumprimento de exigências sanitárias para os mercados mais exigentes. Estudos críticos como em Rowe et al. (2019) discutem, contudo, que o enfoque ainda permanece em ganhos de produtividade, não necessariamente considerando o aspecto de bem-estar animal. No caso de frangos de corte, com ambientes de alta densidade de população e ciclo curto (em média 45 dias), este fato demanda maior atenção pelos agentes envolvidos e tem sido alvo de investigação com base em análise de imagens (OKINDA et al, 2020). É digno de nota que alguns autores chamam à atenção para a consolidação de estratégias existentes antes da adoção de tecnologias disruptivas associadas ao 4.0, caracterizando o conceito intermediário de Indústria 3.5 (WANG et al, 2021).

Neste sentido, de modo a contemplar a diversidade de aplicações, os trabalhos selecionados foram classificados em duas categorias (i) diagnóstico de doenças/bioinformática veterinária (ii) monitoramento de produção e processamento. 
Este trabalho portanto amplia e atualiza o estudo de Milosevic et al. (2019). Dada a atualidade do tema de bioinformática, este tópico foi adicionado à categoria (i) sob o enfoque veterinário. $\mathrm{O}$ termo 'processamento' na categoria (ii) busca contemplar o conceito farm-to-fork, incluindo o controle de qualidade dos produtos.

A Tabela 1 abaixo resume as áreas de aplicação e tarefas baseadas em técnicas de IA, descritos nas subseções a seguir.

Tabela 1: quadro resumo de aplicações, tarefas e trabalhos relacionados

\section{Autores}

A. Banakar et al., 2016

A. Mortensen et al., 2016

M. Kamruzzaman et al., 2016

S. Amraei et al., 2018

S. Barbon et al., 2018

Y. Fajar et al., 2018

N. Perez et al., 2018

X. Zhuang et al., 2018

E. Rowe et al., 2019

B. Milosevic et al., 2019

R. Ribeiro et al., 2019

A. Polewko et al., 2020

C. Okinda et al., 2020

G. Kollenburg et al., 2020

T.L. Passarafo et al., 2020

J. Astill et al., 2020

K. Cuan et al., 2020

R. Yusof et al., 2020

I. Nyalala et al., 2021

D. Seo et al., 2021

C. Wang et al., 2021

S. Yousefinaghani et al., 2021

D. Dourou et al., 2021

J. You et al., 2021

C. Fang et al., 2021
Aplicação

Diagóstico de doenças

Aprimoramento de pesagem

Monitoramento de produção

Predição de peso

Monitoramento de produção

Monitoramento de produção

Monitoramento de produção

Alerta/Diagnóstico de doenças

Ganhos de produtividade no bem-

estar animal

Predição de crescimento e saúde

Aprimoramentos da gestão e manejo de ambiência

Marcadores genéticos

Monitoramento de produção

Monitoramento de produção

Combinação de marcadores

genéticos e rastreabilidade

Diagnóstico de doenças

Diagnóstico de doenças

Monitoramento de produção

Predição de peso e volume de aves

Marcadores genéticos e

rastreabilidade

Módulos para previsões e

monitoramento de peso

Alerta/Diagnóstico de doenças

Monitoramento de produção

Módulos de monitoramento de peso

Diagnóstico de doenças

\section{Tarefa}

Mineração de

dados

Regressão

Regressão

Regressão

Classificação

Mineração de

dados

Mineração de

dados

Classificação

Mineração de

dados

Classificação

Mineração de

dados

Regressão

Classificação

Regressão

Regressão

Regressão

Sons / Regressão

Mineração de

dados

Mineração de

dados

Regressão

Mineração de

dados

Classificação

Classificação

Classificação

Classificação 


\subsection{Diagnóstico de doenças/bioinformática veterinária}

A identificação precoce de condições de gatilho para doenças comuns em aviários a partir de variáveis de ambiência, bem como o monitoramento de comportamento são problemas de pesquisa que fomentam a relação academia-indústria, sendo tais estudos frutos de parcerias ou realizados em instalações próprias de institutos de pesquisa e universidades, reproduzindo as condições reais do setor produtivo em escala adequada. Banakar et al. (2016) propõem um modelo de estudo para o diagnóstico inteligente de doenças aviárias com base em sinais sonoros dos frangos, utilizando um dispositivo para diagnosticar doenças aviárias, como a Influenza Aviária, a Doença de Newcastle (ND) e Vírus da Bronquite (BV), usando métodos de mineração de dados e teoria da evidência DempsterShafer (D-S). Além da vocalização, Astill et al. (2018) revisam estudos de modelos preditivos para detecção de gripe aviária que consideram uma variedade de fontes, com análises de imagens, vídeo e robótica móvel, para inspeção de animais mortos ou doentes, além da limpeza no ambiente. Na mesma linha, Cuan et al. (2020) focam no vírus da gripe aviária H9N2. O método proposto detecta sons complexos emitidos pelas aves baseado nas diferenças espectrais, obtendo um espectrograma para entrada na rede neural convolucional. Numa abordagem diferenciada e baseada em série histórica de dados, Yousefinaghani et al. (2021) definem um framework e dataset com escala espaçotemporal para predição de ocorrência e disseminação em área geográfica, com utilização de features associadas à gripe aviária.

Com o avanço da tecnologia de sensores, equipamentos de detecção, e consequente injeção de dados nos sistemas inteligentes, a antecipação de ações e disparo de alarmes resultante da operação pode ser um diferencial inovador. Por exemplo, Zhuang et al. (2018) apresentam um sistema de alerta baseado em imagens de câmeras e extração de features, para classificar a pose/comportamento de aves saudáveis e doentes. O modelo de Máquina de Vetores de Suporte (SVM) com hiperparâmetros e a função de kernel POLY se destacou, atingindo uma precisão de 99,46\% nas amostras de teste. De modo similar, Fang et al. (2021) comparam frameworks de redes neurais profundas para estimação não invasiva de pose e identificação de diferentes movimentos das aves e associação às enfermidades, com destaque para o classificador Naive Bayes.

A bioinformática enquanto área multidisciplinar com enfoque na determinação de genes, proteínas, e suas relações, encontra atual e relevante aplicação na área veterinária, com especial atenção à avicultura. Visto o volume de dados e relações estatísticas inerentes, técnicas de IA e aprendizagem de máquina têm sido empregadas. A seleção e combinação ótima de marcadores genéticos, para fins de qualidade e tolerância à contaminantes das matrizes (PASSAFARO et al., 2020; POLEWKO-KLIM et al., 2020) e posterior rastreabilidade de origem (SEO et al., 2021) são exemplos de aplicações em crescimento. Destaca-se a parceria de Passafaro et al. (2020) com a empresa CobbVantress, valorizando a interação academia-indústria.

\subsection{Sistemas de monitoramento de produção}

O acompanhamento do ganho de peso diário é determinante para atender aos padrões da indústria, garantindo a remuneração adequada dos agentes envolvidos e qualidade final dos produtos. Existem sistemas de pesagem automática baseados em balanças, imagens ou uma combinação de ambos. Uma ampla revisão sobre técnicas baseadas em imagens, não apenas para frango de corte, pode ser encontrada em Nyalala et al. (2021).

Mortensen et al. (2016), a partir de dataset rotulado com 13.000 imagens para 
treinamento de rede neural Bayesiana, utilizaram câmera 3D para capturar o frango sobre uma balança, obtendo 12 descritores de peso diferentes que permitem estimar o peso individual. Amraei et al. (2018) propõem um sistema equivalente, com dataset de 2440 imagens e combinação da Transformada de Hough (HT) generalizada e método de ChanVese para segmentação. Os 6 descritores obtidos são utilizados para construção de modelo de regressão para predição de peso. Diferentemente, Wang et al. (2021) propõem uma estrutura baseada em dados, incluindo dois módulos para monitoramento e previsão de peso, por meio de análise de big data coletados da nuvem da Amazon (AWS), incluindo sinais eletrônicos coletados das granjas por meio de vários sensores e dispositivos. O módulo de monitoramento de peso é construído por um modelo que integra uma mistura gaussiana, reamostragem de bootstrapping e técnica de média ponderada para estimar o peso atual de frangos de corte vivos na granja. Dada a complexidade em filtrar dados obtidos de balanças automáticas e maximizar sua confiabilidade, You et al. (2021) comparam os algoritmos K-Nearest Neighbors (KNN), Random Forest, SVM e redes neurais para classificação de outliers, obtendo melhores resultados com o Random Forest.

Seja no processamento das carnes no frigorífico (YUSOF et al., 2020), na identificação da qualidade de carne em aves saudáveis para exigências sanitárias regionais ou culturais (FAJAR, 2018), ou na classificação das partes do frango (PEREZ et al., 2018), o uso combinado de câmeras hiperespectrais, para aquisição de dados com IA é comum em vários trabalhos. Kollenburg et al. (2020) investigaram a autenticidade de filés de frango com base em imagens no espectro Infravermelho Próximo (NIR) e técnicas de estatística multivariada, enquanto Kamruzzaman et al. (2016) utilizaram regressão por mínimos quadrados parciais para detecção de adulteração em carne moída de frango. Análise semelhante foi realizada por Barbon et al. (2018) porém com utilização de árvore de decisão. Mais recentemente, a presença de contaminantes no fígado do frango foi investigada por Dourou et al. (2021), com opção pela combinação de árvores de decisão (Extra Trees) para extração de características e SVM para classificação.

\section{Conclusões}

A presente revisão apresentou resultados de pesquisa bibliográfica no período 2016-2021 de sistemas baseados em IA aplicados à avicultura de frangos de corte. Dada a amplitude dos conteúdos dos trabalhos, foi adotada a classificação nas categorias de diagnóstico de doenças/bioinformática veterinária e sistemas de monitoramento de produção, incluindo aspectos de controle de qualidade e processamento agroindustrial.

O diagnóstico antecipado e com assertividade, com indicação de previsão ou probabilidade da ocorrência de doenças em frangos de corte é de grande interesse prático, com abordagens baseadas em monitoramento de comportamento e correlações com a ambiência do aviário. Adicionalmente é notório o avanço nas pesquisas de bioinformática para principalmente o melhoramento genético para minimizar suscetibilidade a doenças. De modo complementar, os sistemas de monitoramento de produção visam otimizar a predição de peso de abate, com estudos de técnicas que possam filtrar medidas de peso indevidas ou incoerentes. Destaca-se o número expressivo de trabalhos na etapa de controle de qualidade da carne de frango, com utilização de câmeras hiperespectrais no espectro NIR em combinação com aprendizagem de máquina. 
A presença da IA é realidade na avicultura industrial de frangos de corte, dado o contexto internacional da pesquisa. Embora ainda caracterizada como um elemento diferenciador, espera-se que a incorporação da IA venha a ser natural no futuro próximo, dada a gama de ferramentas e plataformas disponíveis que abstraem a complexidade de seus algoritmos e facilitam a adoção. Aliada a outras tecnologias habilitadoras 4.0, a saber, IoT, big data e computação em nuvem, tem demonstrado contribuir para a produtividade, com amplo campo de pesquisa e problemas em aberto na cadeia de produção. Dado o estágio de desenvolvimento das grandes empresas avícolas nacionais e os recordes de exportação, a adoção das tecnologias 4.0, em particular a IA, pode estar inserida nos processos frigoríficos e de logística, necessitando de maior adoção pelos avicultores, principalmente para manejo de ambiência e acompanhamento de ganho de peso. Embora os resultados de pesquisa científica com os critérios adotados neste trabalho não apontem para publicações resultantes da relação indústria-academia nacional, fatores como sigilo por propriedade intelectual e priorização por publicação em eventos serão considerados em trabalhos futuros.

\section{Referências}

AMRAEI, S.; MEHDIZADEH, S. A.; NAAS, I. A. Development of a transfer function for weight prediction of live broiler chicken using machine vision. Engenharia Agrícola, v. 38, p. 776-782, 2018.

ASSOCIAÇÃO BRASILEIRA DE PROTEÍNA ANIMAL. Relatório anual 2021. 2021. Disponível em: <https://abpa-br.org/relatorios/>. Acesso em: 18/ago. 2021.

ASTILL, J. et al.. Smart poultry management: smart sensors, big data, and the internet of things. Computers and Electronics in Agriculture, v. 170, 105291, 2020.

ASTILL, J. et al. Detecting and predicting emerging disease in poultry with the implementation of new technologies and big data. Frontiers in Veterinary Science, v. 5, 263, 2018.

BANAKAR, A.; SADEGHI, M.; SHUSHTARI, A. An intelligent device for diagnosing avian diseases: Newcastle, infectious bronchitis, avian influenza. Computers and Electronics in Agriculture, v. 127, p. 744-753, 2016.

BARBON, S. et al. Machine Learning Applied to Near-Infrared Spectra for Chicken Meat Classification. Journal of Spectroscopy, v. 2018, 2018.

BASSI, N. S. S.; SILVA, C. L. Oportunidades e desafios para a cadeia produtiva de frangos de corte brasileira. Avicultura Industrial, Itu, ed. 1271, ano 109, n. 10, p.16-21, 2017.

BASSI, N. S. S.; SILVA, C. L. Pesquisa e Desenvolvimento na Cadeia Produtiva de Frangos de Corte no Brasil. Revista de Economia e Sociologia Rural [online], v. 56, n. 3, p. 467-482, 2018.

BRASIL. Agência Brasileira de Desenvolvimento Industrial. ABDI divulga selecionados do Edital Agro 4.0. 2020a. Disponível em: <https://www.abdi.com.br/postagem/abdidivulga-selecionados-do-edital-agro-4-0>. Acesso em: 18 ago. 2021.

BRASIL. Financiadora de Estudos e Projetos. Seleção pública MCTI/FINEP/FNDCT. Subvenção econômica à inovação - tecnologias 4.0, 2020b. Disponível em: $<$ http://www.finep.gov.br/chamadas-publicas/chamadapublica/643>. Acesso em: 22 ago. 2021. 
CUAN, K. et al. Detection of avian influenza-infected chickens based on a chicken sound convolutional neural network. Computers and Electronics in Agriculture, v. 178, id. 105688, 2020.

DOUROU, D. et al. Rapid microbial quality assessment of chicken liver inoculated or not with salmonella using FTIR spectroscopy and machine learning. Frontiers in Microbiology, v. 11, 2021.

FAJAR, Y. Artificial neural network for healthy chicken meat identification. Int. Journal of Artificial Intelligence, v. 7, n. 1, p. 63-70, 2018.

FANG, C. et al.. Pose estimation and behavior classification of broiler chickens based on deep neural networks. Computers and Electronics in Agriculture, v. 180, id. 105863, 2021.

KAMRUZZAMAN, M., MAKINO, Y., OSHITA, S. Rapid and non-destructive detection of chicken adulteration in minced beef using visible near-infrared hyperspectral imaging and machine learning. Journal of food engineering, v. 170, p. 8-15, 2016.

KOLLENBURG, G. et al. Dataset of the application of handheld NIR and machine learning for chicken fillet authenticity study. Data in Brief, v. 29, 2020.

MILOSEVIC, B. et al. Machine learning application in growth and health prediction of broiler chickens. World's Poultry Science Journal, v. 75, p. 401-410, 2019.

MORTENSEN, A. K.; LISOUSKI, P.; AHRENDT, P. Weight prediction of broiler chickens using 3D computer vision. Computers and Electronics in Agriculture, v. 123, p. 319326, 2016.

MOTHER, D. et al. Preferred reporting items for systematic reviews and meta-analysis: the PRISMA statement. PLoS Med, 6, e1000097, 2009.

NYALALA, I. et al. Weight and volume estimation of poultry and products based on camera vision systems: a review. Poultry science, v. 100, n. 5, id. 101072, 2021.

OKINDA, C. et al. A review on computer vision systems in monitoring of poultry: a welfare perspective. Artificial Intelligence in Agriculture, v. 4, p. 184-208, 2020.

OLIVEIRA, F. D.; SANTANA, A.; PEREIRA, L. F. A. Impactos da IoT na Avicultura: um Mapeamento Sistemático. In: XII Congresso Brasileiro de Agroinformática, Indaiatuba, p. 418-427, 2019.

PASSAFARO, T. L. et al. Would large dataset sample size unveil the potential of deep neural networks for improved genome-enabled prediction of complex traits? the case of broiler weight prediction. BMC Genomics, v. 21, 771, 2020.

PEREZ, N. et al. Classification of Chicken Parts Using a Portable Near-Infrared (NIR) Spectrophotometer and Machine Learning. Applied spectroscopy, v. 72, n. 12, p.17741780, 2018.

POLEWKO-KLIM, A. et al. Sensitivity analysis based on random forest machine learning algorithm identifies candidate genes for regulation of innane and adaptive immune response of chicken. Poultry Science, v. 99, n. 12, p. 6341-6354, 2020.

RIBEIRO, R. et al. Generating action plans for poultry management using artificial neural networks. Computers and Electronics in Agriculture, v. 161, p. 131-140, 2019. 
ROCHA, G. B.; SILVA, I. J. O. A dinâmica da Zootecnia de Precisão e tecnologias de suporte atuais. 2020. Disponível em: $<$ https://www.aviculturaindustrial.com.br/imprensa/a-dinamica-da-zootecnia-deprecisao-e-tecnologias-de-suporte-atuais/20201218-085415-w971>. Acesso em: 18 ago. 2021.

ROWE, E.; DAWKINS, M.S.; GEBHARDT-HENRICH, S.G. A systematic review of precision livestock farming in the poultry sector: is technology focussed on improving bird welfare? Animals, v. 9, 614. doi:10.3390/ani9090614, 2019.

SANTANA, L.; OLIVEIRA, J. Agricultura 4.0 e o desenvolvimento de pesquisas de computação aplicada às ciências agrárias. SBC Horizontes, marco. 2021. ISSN 21759235. Disponível em: <http://horizontes.sbc.org.br/index.php/2021/03/Agricultura-4-0e-o-desenvolvimento-de-pesquisas-de-computacao-aplicada-as-ciencias-agrarias $/>$.

Acesso em: 18 ago. 2021.

SCHWAB, K. The fourth industrial revolution. World Economic Forum, 2016.

SEO, D. et al. Identification of target chicken populations by machine learning models using minimum number of SNPs. Animals, v. 11, n. 241, 2021.

WANG, C.; CHEN, Y.; CHIEN, C. Industry 3.5 to empower smart production for poultry farming and an empirical study for broiler live weight prediction. Computers \& Industrial Engineering, v. 151, id. 106931, 2021.

YOUSEFINAGHANI, S. et al. A framework for the risk prediction of avian influenza occurrence: An Indonesian case study. PLoS ONE, doi: https://doi.org/10.1371/journal.pone.0245116, 2021.

YUSOF, R. et al. Image segmentation and verification based on machine learning for vision inspection of chicken slaughtering. Journal of Physics, v. 1147, n. 1, 2020.

YOU, J. et al. A supervised machine learning method to detect anomalous real-time broiler breeder body weight data recorded by a precision feeding system. Computers and Electronics in Agriculture, v. 185, 106171, 2021.

ZHUANG, X. et al. Development of an early warning algorithm to detect sick broilers. Computers and Electronics in Agriculture, v. 144, p. 102-113, 2018. 